\title{
Performance Analysis of S-SFR-based OFDMA Cellular Systems
}

\author{
Yi-Kang Kim¹, Choong-Ho Cho ${ }^{1}$, Seok-Ho Yoon ${ }^{1}$ and *Seung-Yeon Kim ${ }^{1}$ \\ ${ }^{1}$ Department of Computer Convergence Software, \\ Korea University, Sejong, Korea \\ [e-mail: \{kimyikang, chcho, bluepig5, kimsy8011\}@korea.ac.kr] \\ *Corresponding author: Seung-Yeon Kim
}

Received April 3, 2018; revised May 22, 2018; revised June 28, 2018; accepted July 5, 2018; published January 31, 2019

\begin{abstract}
Intercell interference coordination (ICIC) is considered as a promising technique to increase the spectral efficiency of OFDMA cellular systems. The soft frequency reuse (SFR) and fractional frequency reuse (FFR) are representative and efficient management techniques for ICIC. Herein, to enhance the performance of the SFR scheme, we propose a call admission (CAC) scheme. In this CAC scheme, called Spectrum handoff-SFR(S-SFR), the spectrum handoff technique is applied to the user equipment (UE) located near the cell center. We derive the traffic analysis model to describe the S-SFR. In addition, a two-dimensional (2-D) Markov chain and an outage analysis are used in our analytical model. From the traffic analysis, the significant performance measures are the outage probability, call blocking probability, system throughput and resource utilization. Based on those, the outage probability and system throughput are obtained using resource utilization as an interference pattern. The analytical results are verified with computer simulation results. Finally, we compare our proposed scheme with other ICI schemes.
\end{abstract}

Keywords: Spectrum handoff, intercell interference coordination(ICIC), call admission control(CAC), 2-D Markov chain, soft frequency reuse(SFR)

This work was supported by the National Research Foundation of Korea(NRF) grant funded by the Korea government (NRF-2017R1C1B5017797). 


\section{Introduction}

\subsection{Background}

Current and emerging wireless cellular systems (i.e., LTE-A, WiFi, 5G) characterize more dense base station (BS) deployments providing higher network capacity as mobile user traffic increases. Orthogonal frequency division multiple access (OFDMA), which combined scheduling and resource allocation techniques, is the most common way to achieve high system performance [1] and [2]. In OFDMA-based networks, the orthogonality between subcarriers renders the intracell interference negligible. However, because these networks are targeting a frequency reuse factor of 1 (FRF 1), which refers to reusing the whole spectrum in each cell, it also causes high intercell interference (ICI), particularly in cell edge areas. The performance of wireless cellular networks may drop dramatically due to the impact of high ICI. Therefore, ICI coordination (ICIC) is a significant issue in the design of cellular systems, especially 4G and 5G systems [3].

To overcome ICI, various ICIC schemes have been studied in the literature under various spectrum conditions and network structures. In [4], the authors classify the ICIC schemes along several dimensions to differentiate the schemes between static versus adaptive and centralized versus coordinated. Static ICIC is based on frequency reuse (FR) schemes such as frequency reuse factor 3 (FRF 3) [5]. Adaptive FR that does not require cooperation between BSs is based on the adaptive resource and/or power allocation schemes, such as soft frequency reuse (SFR) and fractional frequency reuse (FFR) [6]-[8]. A centralized scheme is operated through a central control entity that manages multiple BSs simultaneously [9][10]. Finally, coordinated distributed schemes utilize the specific indicators exchanged between the neighboring BSs via the X2 interface for reducing ICI [11]. Among the various ICIC schemes mentioned above, FFR and SFR are considered as an attractive technique for state-of-the art OFDMA-based cellular systems. However, there is limited prior research on the performance analysis through traffic models in FFR and SFR. So, we consider the performance analysis through a traffic model in adaptive FR schemes as well as a call admission control (CAC) algorithm.

Fig. 1 shows an example of frequency assignment for FFR and SFR as the adaptive FR schemes. As shown, a cell is divided into two regions: the inner region and the outer region. In both schemes, the users in the outer region can be assigned a different frequency sub-band with the adjacent cells. As shown in Fig. 1, for the inner region in FFR, the users in that region can use the frequency sub-band in the manner of FRF 1 in the neighboring cells (Fig. 1 (a)) while, in SFR, the users can use the whole frequency band and share the bandwidth with the neighboring cells as FRF 1 (Fig. 1 (b)). From the FFR scheme, the users in the inner region can avoid the interferences by the signals for the users in the outer region of the neighboring cell. For the users in the outer region, they receive the interfering signals from the neighboring cell using the same frequency sub-band. Thus, the number of interfering signals received from the neighboring cells is decreased. However, since the frequency sub-band is limited, when the resources are heavily utilized, the FFR scheme may result in high call blocking probability, which is defined as the ratio of the number of calls lost in the system to the total number of newly initiated calls [23]. For the SFR scheme, as the users in the inner region can use the whole frequency band compared with the FFR scheme, a call blocking probability of SFR is decreased compared to that of FFR. In addition, the user in the outer region of SFR may 
undergo the interference by signal for the user in the inner region of the neighboring cell. Accordingly, we consider an SFR scheme to maximize the system throughput, while maintaining some quality of service (QoS) constraints. More specifically, we propose a CAC algorithm with a preemption technique in SFR to reduce the call blocking probability and improve the system throughput.
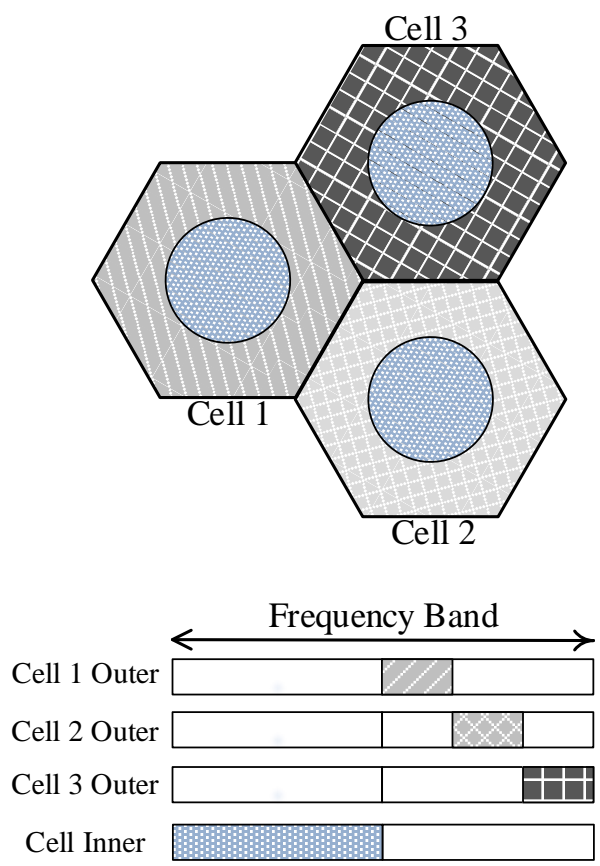

(a) Fractional Frequency Reuse (FFR)
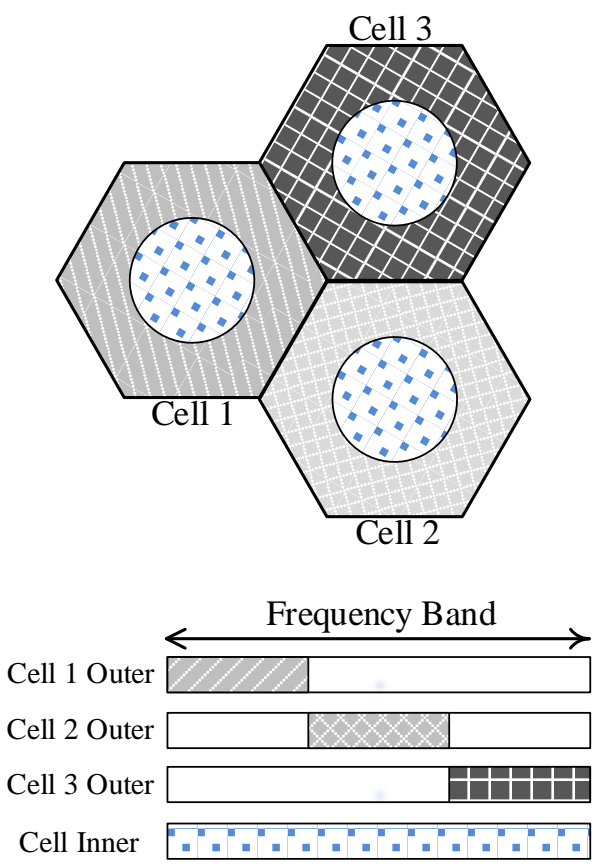

(b) Soft Frequency Reuse (SFR)

Fig. 1. Example of frequency assignment for FFR and SFR schemes

\subsection{Related Work}

To effectively coordinate ICI and improve the spectrum efficiency, many studies on the performance of FFR and/or SFR systems has been reported [12]-[25]. In [12] and [13], Novlan and Zhu et al. analyzed the FFR and SFR performances considering the two types of FRs, depending on the inner and outer regions. In [14], the authors showed the improved performance of the outage probability and the average cell-edge user throughput through coordinated SFR (CoSFR), in which the multicell networks with nonuniform user distribution was considered. In [15], the authors developed a model for analyzing FFR-aided OFDMAbased multi-cellular networks that incorporates shadowing effects and considers the irregular cellular networks. In [24], the authors proposed an analytical model for evaluating the performance of FFR-aided heterogeneous networks (HetNet) that considers both co-tier and cross-tier interference. [25] developed a framework for allowing the design of optimal 5th-percentile user rate constrained FFR/SFR-aided multi-cellular OFDMA-based networks using channel-aware PF scheduling. In [16] and [17], the FFR and/or SFR schemes were applied to heterogeneous cellular networks, and the coverage probability for these schemes was derived under the Rayleigh fading. In [22], to maximize the overall network throughput and minimize the outage probability, the authors proposed a resource allocation and power control scheme in FFR-based heterogeneous cellular networks, in which the limiting the cross-tire interference below a predefined threshold. [18] studied a global energy efficiency 
optimization algorithm in multicell networks with multiple users, in which the convex optimization method was considered. Additionally, in [19], to minimize network interference, the authors established the multilayer SFR scheme combined with cell sectoring where the spectrum is allocated intelligently among several regions. Literatures that focus on the analysis of SFR with the traffic model are limited. In [21], the authors proposed a CAC using the one-dimensional Markov chain model in FFR-based cellular systems to improve the performance of call blocking probabilities and resource utilizations. [7] and [20] developed an analytical model to describe the CAC in SFR-based systems and characterized the outage probability and/or new call blocking probability. [7] models a 3-D Markov chain for traffic analysis, while our proposed model uses a 2-D Markov chain. Specifically, in [7], SFR causes a forced termination problem of an ongoing call. In this paper, we propose a method to overcome the forced termination problem.

\subsection{Primary Contributions}

In this research, we propose the Spectrum handoff-SFR (S-SFR) under a CAC algorithm with a preemption technique to enhance the performance of OFDMA cellular network. From the modeling point of view, we herein consider the small-scale fading as in [12]-[19]. In contrast to [12]-[19], we will furthermore consider a traffic model as well as a CAC algorithm. In addition, we consider the outage analysis using the interference patterns. The four key contributions in our paper are summarized as follows:

1. A CAC algorithm is proposed, in which a preemption technique is applied to the user equipment (UE) in the inner zone. In contrast to [7], we model a 2-D Markov chain for the traffic analysis of the proposed scheme-based cellular systems, in which we assume that the call interarrival time and call duration are exponentially distributed. In the analysis, the call blocking probabilities for users and resource utilization are obtained. These are used for some design criteria, such as the inner region size, to maximize the system throughput.

2. We derive a model of the signal to interference and noise ratio (SINR) for a user by considering the cellular geometry, ICI, and fading effects. This paper, in contrast to [12]-[22], assumes that some neighboring cells statistically generate ICIs, depending on the resource utilization of each cell.

3. The system throughput is defined, in which the QoS pertains to the call blocking of user calls and the outage probabilities. In other words, the system throughput can be obtained from the effective carried load.

4. We compare the performance with the different adaptive FR schemes by differentiating the inner region size and the traffic load, where the FFR and SFR schemes are considered. Our proposed scheme outperforms the other schemes with regard to the system throughput based on some criteria.

The remainder of this paper is organized as follows: Section 2 introduces the system model with the proposed scheme, our assumptions, and an optimization problem for designing this system. In section 3, we analyze this system to solve our optimization problem. In section 4, we discuss the numerical studies and present some concluding remarks in section 5 . 


\section{System Model}

\subsection{Cellular network topology}

We consider a cellular network with a Spectrum handoff-SFR(S-SFR)-based system. We consider the 2-tier cell cluster model used in many inter-cell interference studies [12-22]. As shown in Fig. 2, each eNodeB (eNB) is located at the cell center, in which the length of one side of a hexagonal cell is $R$. When the cell is deployed in 2-tier around the target cell, the number of cells in the system model is 19 . The radius of a circular cell whose area is the same as a hexagonal cell $R_{0}$ is given by $\sqrt{3 \sqrt{3} R^{2} / 2 \pi}$. Each cell is further divided into two regions: the inner region of radius $Z$ and the outer region, which is outside the inner region. Herein, the UE located in the inner and the outer regions are called the inner UE and outer UE, respectively. As a more realistic model, to determine whether a UE is in the inner region or the outer region, the eNB can use the received signal strength (RSS), which is measured and then reported by the UE to estimate the distance.

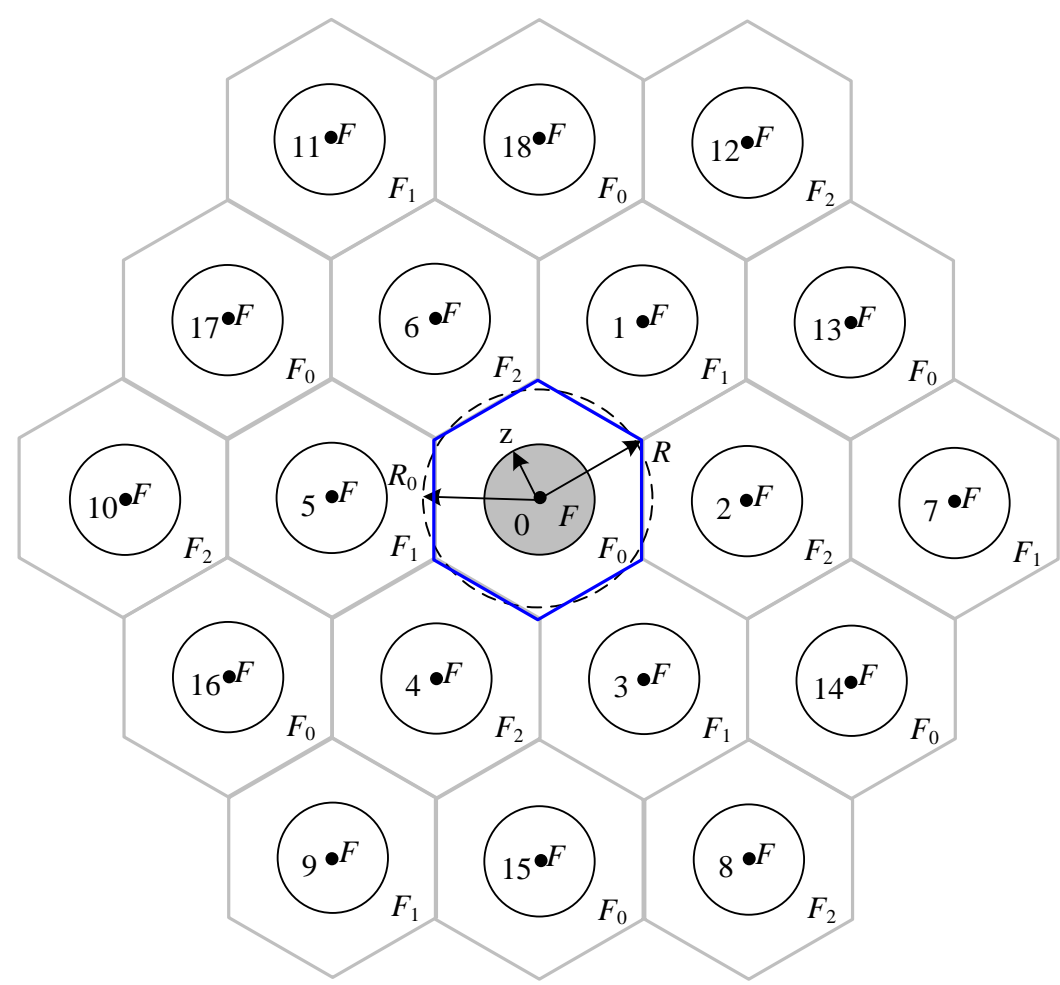

Fig. 2. Network topology for S-SFR-based cellular systems

In our model, we assume that the reuse factor of 3 is used in our S-SFR-based cellular system, and one set of frequency physical resource block (PRB), $F_{i}$, is assigned to each of the outer regions of the 19-cell cluster in a regular pattern, as shown in Fig. 2. As [7], the UE in the inner region (inner UE) may use an available PRB in $F$ while those in the outer region (outer $\mathrm{UE}$ ) may use any available PRB only in the outer PRB, $F_{i}, i=0,1,2$. Suppose that the outer UE on the tagged cell use the PRBs in $F_{0}$. Subsequently, the PRBs in $F_{1}$ and $F_{2}$ are assigned alternately to outer UE in the odd- and even-numbered first-tier cells, respectively. For the 
second-tier cells, the PRBs of $F_{0}$ are assigned to the outer UE in cells numbered from 13 to 18 in Fig. 2, while the PRBs of $F_{1}$ and $F_{2}$ are assigned alternately to the outer UE in cells numbered from 7 to 12 . As shown in Fig. 2, the 18 neighboring cells are divided into three sets based on the distance from the target cell, $\mathrm{eNB}_{0}$. The first set of cells is the six neighboring cells numbered from 1 to 6 in the first tier, which are $d_{1}=\sqrt{3} R$ away from the center of the target cell. The second set includes the six cells in the second tier, which are numbered from 7 to 12 . These cells are $\mathrm{d}_{2}=2 \mathrm{~d}_{1}$ away from eNB $\mathrm{B}_{0}$. The third set is the set containing the remaining six cells in the second-tier cells, which are numbered from 13 to 18 and adjacent to the $d_{1}$ cells and $d_{2}$ cells. These are $d_{3}=3 R$ away from $e B_{0}$.

\subsection{Call admission control of S-SFR}

For traffic modeling, a number of UEs can initiate multiple calls when trying to occupy radio resources, where the basic unit of radio resources is referred to as the PRB. We assume that each of the eNBs has a total of $C$ PRBs, which consists of $C_{i}$, inner PRBs and $C_{o}$, outer PRBs, where $C_{i}=C-C_{o}$. We also assume that a UE shall access to its serving eNB, whose access interval follows an exponential distribution with mean $1 / \lambda$ (sec/call), and the UEs are uniformly distributed over the cell. Let $\omega$ be the fraction of the area for the inner region to the cell coverage, i.e., $\omega=\left(z / R_{0}\right)^{2}$. The new call arrival rates of the inner and outer UE are assumed to be $\lambda_{i}=\omega \lambda$ and $\lambda_{o}=(1-\omega) \lambda$, respectively. Each user who successfully receives a PRB shall hold it for exponentially distributed random times with mean $1 / \mu$ (sec).

When a new inner UE is originated, it will first attempt to occupy an inner PRB. If one or more inner PRBs are available, it will be admitted. If no inner PRB is available, it will attempt to occupy an outer PRB. It will be blocked once no outer PRBs are available; this event is called an inner call blocking. When an inner UE occupying an inner PRB is released, an ongoing inner UE occupying the outer PRB is reconnected using a released PRB. This is called spectrum handoff. Therefore, an inner UE can use either an inner or outer PRB if they are available.

For a newly arrived outer UE, it will first attempt to occupy an outer PRB. At this time, if all outer PRBs are in use by the ongoing outer UE, it will be blocked. This is called an outer call blocking. If at least one outer PRB is available, it will be admitted. If no outer PRBs are available but one or more outer PRBs are occupied by the ongoing inner UEs, one of the inner UEs will be randomly chosen and forced to release the outer PRB that it is occupying. The released PRB is then assigned to the newly arrived outer UE. The probability that the inner UE is forcibly terminated is referred to as forced termination probability.

\subsection{Signal to Interference plus Noise Ratio Model}

For a physical-layer modeling, the average power received at a UE from a eNB $j$, i.e., $\operatorname{eNB}_{j}$, is expressed as

$$
P_{j}=P_{t} r_{j}^{-\alpha} h_{j}
$$


where $P_{t}$ is the transmission power of eNB, and $h_{j}$ indicates the small-scale fading, which is assumed to be exponentially distributed with mean $\delta_{j}$, i.e., Rayleigh fading. $r_{j}^{-\alpha}$ denotes a path-loss at a distance $r_{j}$ between $\mathrm{eNB}_{j}$ and a $\mathrm{UE}$ in $\mathrm{eNB}_{0}$ where $\alpha$ denotes the path-loss exponent.

Based on Eq. (1), the SINR of the average power received at a UE from the interference set $\partial$ with $n_{I}$ interfering signals is as follows:

$$
S I N R=\frac{P_{t} r_{o}^{-\alpha} h_{o}}{\sum_{j \in \partial\left(n_{I}\right)} P_{t} r_{j}^{-\alpha} h_{j}+\sigma^{2}},
$$

where $\sigma^{2}$ denotes additive noise.

\subsection{S-SFR Problem Formulation}

The system throughput denoted by $S_{t h}$ is obtained from the effective carried load. Accordingly, for a given Erlang load, which is defined as the number of calls per unit call holding time, $\vartheta=\lambda / \mu$, and $z, S_{t h}$ can be expressed as

$$
S_{t h}(\vartheta, z)=\left[\lambda_{i}\left(1-P_{B i}\right)\left(1-P_{f}\right)\left(1-P_{o i}\right)+\lambda_{o}\left(1-P_{B o}\right)\left(1-P_{o o}\right)\right] / \mu,
$$

where $P_{B i}$ and $P_{B o}$ are the call blocking probabilities of the inner and outer UE, respectively; $P_{f}$ denotes the forced termination probability. In addition, $P_{o i}$ and $P_{o o}$ are the outage probabilities of the inner and outer UE, respectively. It is noteworthy that $S_{t h}$ depends on Erlang load $\vartheta$ and $z$. Thus, an optimization problem for $S_{t h}$ can be formulated as follows:

$$
\begin{array}{ll}
\text { Max } & S_{t h}(\vartheta, z), \\
\text { Subject to } & P_{B i}, P_{\text {Bo }} \leq \varepsilon_{B} \text { and } P_{o} \leq \varepsilon_{o}, \\
\text { Variables } & \vartheta, z,
\end{array}
$$

where $\varepsilon_{B}$ denotes the threshold of the call blocking probability, $\varepsilon_{o}$ denotes the tolerance of the outage probability. The call blocking probability and the outage probability will be derived in the next section.

\section{Performance Analysis}

\subsection{Traffic analysis}

Assuming the characteristics of traffic, the process of PRB occupation can be modeled as a continuous-time Markov chain. For the CAC of S-SFR, the state transition diagram is described by an integer pair $\left(n_{i}, n_{o}\right)$ as shown in Fig. 3, where $n_{i}$ and $n_{o}$ denote the number of inner UE and outer UE, respectively. As the outer UE have the priority to use the outer PRB, 
the inner UE using the outer PRB can be preempted by the outer UE. Depending on the existence of inner UEs occupying the outer PRB, a forced termination in state $\left(n_{i}, n_{o}\right)$ can move the state to $\left(n_{i}-1, n_{o}+1\right)$, where $n_{i}$ is greater than $C_{i}$. This is because only the inner UE occupy the outer PRB by spectrum handoff.

Let $P\left(n_{i}, n_{o}\right)$ be the steady-state probability mass function (PMF). $P\left(n_{i}, n_{o}\right)$ satisfies the following balance equations for $0<n_{i}<C$ and $0<n_{o}<C_{o}$ :

$$
\begin{aligned}
P\left(n_{i}, n_{o}\right)\left(\lambda_{i}+\lambda_{o}+n_{i} \mu+n_{o} \mu\right) & =\lambda_{i} P\left(n_{i}-1, n_{o}\right)+\lambda_{o} P\left(n_{i}, n_{o}-1\right) \\
& +\left(n_{i}+1\right) \mu P\left(n_{i}+1, n_{o}\right)+\left(n_{o}+1\right) \mu P\left(n_{i}, n_{o}+1\right) .
\end{aligned}
$$

Subsequently, the other balance equations can be visualized by the state-transition flow diagram shown in Fig. 3. $P\left(n_{i}, n_{o}\right)$ can be found by solving the balance equations together with the normalization condition,

$$
\sum_{n_{i}} \sum_{n_{o}} P\left(n_{i}, n_{o}\right)=1
$$

Once $P\left(n_{i}, n_{o}\right)$ is obtained, we can obtain the following performances.

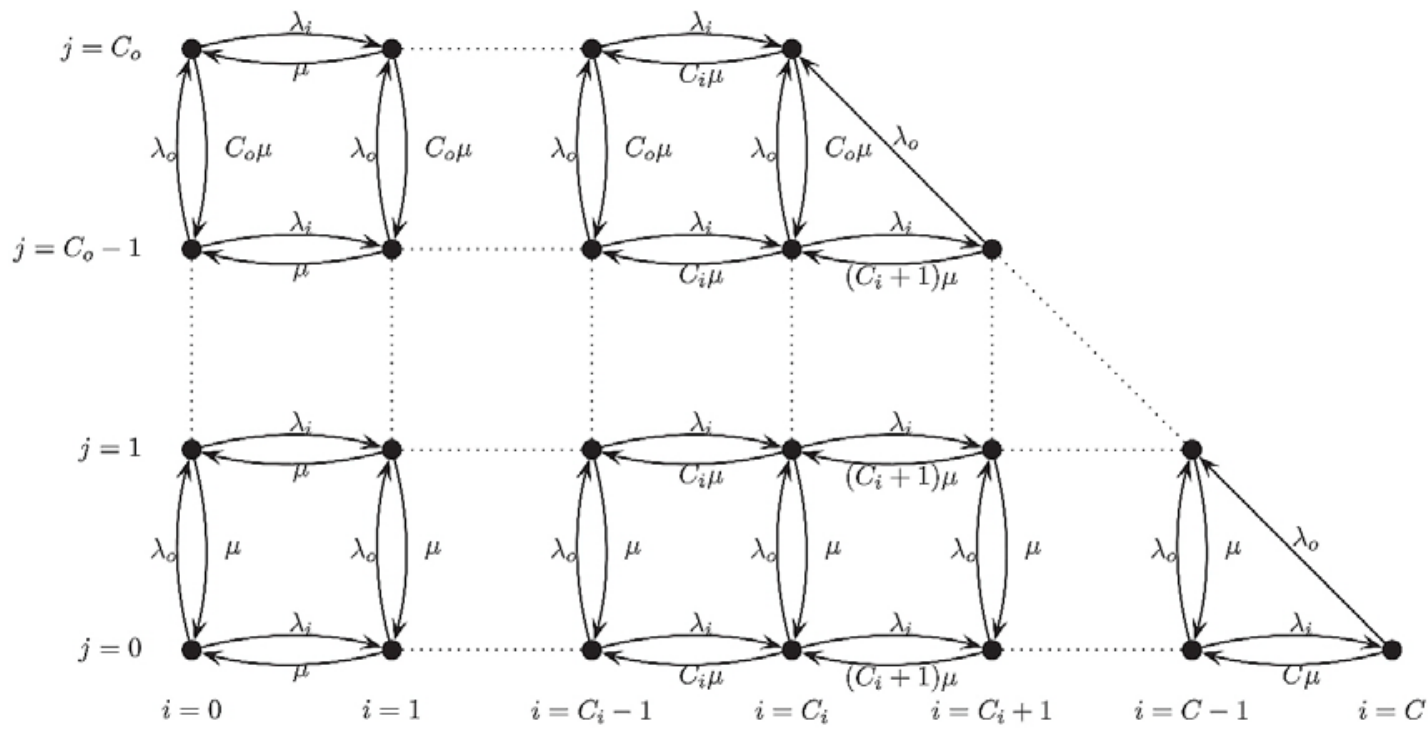

Fig. 3. The two-dimensional Markov chain model for S-SFR

\subsubsection{Call blocking probability}

From $P\left(n_{i}, n_{o}\right)$, the call blocking probability of inner UE, $P_{B i}$, and that of the outer UE, $P_{B o}$, are, respectively, obtained by 


$$
P_{B i}=\sum_{n_{i}=C_{i}}^{C} P\left(n_{i}, C-n_{i}\right), \quad P_{B o}=\sum_{n_{i}=0}^{C_{i}} P\left(n_{i}, C_{o}\right) .
$$

From Eq. (7), we can obtain the aggregate call blocking probability, $P_{B}$, as follows:

$$
P_{B}=\omega P_{B i}+(1-\omega) P_{B o}
$$

\subsubsection{Forced termination probability}

The forced termination probability, $P_{f}$, is considered as the total UE forced termination rate divided by the total UE connection rate. Therefore, it is given as follows:

$$
P_{f}=\frac{\lambda_{o} / \mu \sum_{n_{i}=C_{o}+1}^{C} P\left(n_{i}, C-n_{i}\right)}{\lambda / \mu\left(1-P_{B}\right)} .
$$

\subsubsection{Resource utilization}

To obtain the resource utilization, we first find the mean number of the inner UE and outer UE in the target cell, which are denoted by $\overline{N_{i}}$ and $\overline{N_{o}}$, respectively, and obtained by

$$
\begin{aligned}
& \overline{N_{i}}=\mathrm{E}\left[N_{i}\right]=\sum_{n_{i}=0}^{C_{i}} n_{i} \sum_{n_{o}=0}^{C_{o}} P\left(n_{i}, n_{o}\right)+\sum_{n_{i}=C_{i}+1}^{C} n_{i} \sum_{n_{o}=0}^{C_{o}-n_{i}} P\left(n_{i}, n_{o}\right), \\
& \overline{N_{o}}=\mathrm{E}\left[N_{o}\right]=\sum_{n_{o}=0}^{C_{o}} n_{o} \sum_{n_{i}=0}^{C-n_{o}} P\left(n_{i}, n_{o}\right) .
\end{aligned}
$$

They can also be obtained as follows:

$$
\overline{N_{i}}=\lambda_{i}\left(1-P_{B i}\right)\left(1-P_{f}\right), \overline{N_{o}}=\lambda_{o}\left(1-P_{\text {Во }}\right) .
$$

It is noteworthy that $\overline{N_{i}}$ includes not only the inner UE using the inner PBR, $\overline{N_{i i}}$, but also the inner UE using the outer PBR, $\overline{N_{i o}}$. Thus, the following are obtained:

$$
\begin{aligned}
& \overline{N_{i i}}=\sum_{n_{i}=0}^{C_{i}} n_{i} \sum_{n_{o}=0}^{C_{o}} P\left(n_{i}, n_{o}\right)+\sum_{n_{i}=C_{i}+1}^{C} C_{i} \sum_{n_{o}=0}^{C_{o}-n_{i}} P\left(n_{i}, n_{o}\right), \\
& \overline{N_{i o}}=\overline{N_{i}}-\overline{N_{i i}} .
\end{aligned}
$$

From Eq. (12) and (13), given that a PRB is assigned to an inner UE, the probability that the $\mathrm{PRB}$ is an inner PRB, $v_{i}$, is obtained by 


$$
v_{i}=\frac{\overline{N_{i i}}}{\overline{N_{i}}} .
$$

Additionally, an outer PRB assigned to the inner UE can be used until this UE is forced terminated by a new arriving outer UE. Thus, the time used by the inner UE is given by

$$
\overline{N_{i o_{-} f}}=\underbrace{\left[\lambda_{i}\left(1-P_{B i}\right) P_{f}\right]}_{\text {(a) }} \times \underbrace{\left(\mu-\lambda_{o}\right)}_{\text {(b) }},
$$

where (a) means the mean number of the inner UE forced terminated by the outer UE and (b) means the time used by each forced terminated inner UE. From Eq. (12), (13) and (15), we can obtain the resource utilization of the inner PRB, $\rho_{i}$, and that of the outer PRB, $\rho_{o}$, respectively, as follows:

$$
\rho_{i}=\frac{\overline{N_{i i}}}{C_{i}}, \quad \rho_{o}=\frac{\overline{N_{o}}+\overline{N_{i o}}+\overline{N_{i o_{-} f}}}{C_{o}} .
$$

As mentioned earlier, $\rho_{i}$ and $\rho_{o}$ are used as statistically generated interferences from some neighboring eNBs. From Eq.(16), the total resource utilization, $\rho$, is given by

$$
\rho=\frac{\rho_{i} \cdot C_{i}+\rho_{o} \cdot C_{o}}{C}
$$

Table 1. Summary for interference scenarios

\begin{tabular}{|c|c|c|}
\hline PRB used cases & Utilization & Cell Number \\
\hline \hline \multirow{2}{*}{ Inner PRB used } & $\rho_{i}$ & $2,4,6,8,10,12,13,14,15,16,17,18$ \\
\cline { 2 - 3 } & $\rho_{o}$ & $1,3,5,7,9,11$ \\
\hline \multirow{2}{*}{ Outer PRB used } & $\rho_{i}$ & $1 \sim 12$ \\
\cline { 2 - 3 } & $\rho_{o}$ & $13 \sim 18$ \\
\hline
\end{tabular}

\subsection{Outage Analysis}

To obtain the outage probability for a given threshold $\gamma$ and interference set, $\partial\left(n_{I}\right)$, we first find the complementary outage probability, $P_{o}^{C}\left[\gamma \mid \partial\left(n_{I}\right)\right]$. This can be expressed as

$$
\begin{aligned}
P_{o}^{C}\left[\gamma \mid \partial\left(n_{I}\right)\right] & =\operatorname{Pr}\left[\operatorname{SINR}>\gamma \mid \partial\left(n_{I}\right)\right] \\
& =\int_{0}^{2 \pi} \int_{r_{\min }}^{r_{\max }} \underbrace{\operatorname{Pr}\left[\frac{P_{t} r_{o}(r, \theta)^{-\alpha} h_{o}}{\sum_{j \in\left(n_{I}\right)} P_{t} r_{j}(r, \theta)^{-\alpha} h_{j}+\sigma^{2}}>\gamma\right]}_{\text {(c) }} f_{p}(r, \theta) d r d \theta,
\end{aligned}
$$


where $(r, \theta)$ is the polar coordinated UE in the target eNB, i.e., $\mathrm{eNB}_{0}$ and $f_{p}(r, \theta)$ denotes the joint probability density function (PDF) of $(r, \theta)$, given by

$$
f_{p}(r, \theta)=\frac{1}{2 \pi} \times \frac{2 r}{R_{0}^{2}}, \quad r \leq R_{0}, 0 \leq \theta \leq 2 \pi
$$

Because two regions exist, in Eq. (18), when the inner UE is considered, $r_{\min }$ and $r_{\max }$ are $0^{+}$and $z$, respectively. For the outer UE, they are $z$ and $R_{0}$, respectively. Additionally, the interference set, $\partial$, denotes a set of all active eNBs, which depend on the resource utilization of each eNB. For example, suppose that a UE is using an inner PRB $F_{1}$. Two types of interference scenarios exist: (i) the interferences occur from cells 2, 4, 6, 8, 10, 12, 13, 14, 15, 16,17 , and 18 with resource utilization of inner PRB, $\rho_{i}$, (ii) the interferences occur from cells $1,3,5,7,9$, and 11 with $\rho_{o}$. Similarly, for the case where the UE uses the outer PRB, the interference scenarios are summarized in Table $\mathbf{1}$. We assume that $\rho_{i}$ and $\rho_{o}$ are used as statistically generated interferences from some neighboring eNBs. Thus, the probability that for a UE using an inner PRB, where there are $n_{I}$ interferences, which are $n_{I_{-} i}$ interferences from the inner PRB and $n_{I_{-} o}$ interferences from the outer PRB, $\operatorname{Pr}\left[\partial\left(n_{I}\right): n_{I_{-} i}, n_{I_{-} o}\right]$, is

$$
\left(\begin{array}{c}
12 \\
n_{I_{-} i}
\end{array}\right) \rho_{i}^{n_{I_{-} i}}\left(1-\rho_{i}\right)^{12-n_{I_{-} i}} \times\left(\begin{array}{c}
6 \\
n_{I_{-} o}
\end{array}\right) \rho_{o}^{n_{I_{-} o}}\left(1-\rho_{o}\right)^{6-n_{I_{-} o}} .
$$

Based on 2-3, we can rewrite (c) in Eq. (18) as follows:

$$
(\mathrm{c})=\operatorname{Pr}\left[h_{o}>\gamma\left(\sum_{j \in \partial\left(n_{I}\right)} d_{j}(r, \theta)^{-\alpha} h_{j}\right)+\gamma \sigma^{2} p_{t}^{-1} r_{o}(r, \theta)^{\alpha}\right] \text {, }
$$

where $d_{j}(r, \theta)^{-\alpha}=P_{t} r_{j}(r, \theta)^{-\alpha} / P_{t} r_{o}(r, \theta)^{-\alpha}$. Let $\mathrm{A}=\gamma \sigma^{2} p_{t}^{-1} r_{o}(r, \theta)^{\alpha}$. Therefore,

$$
\begin{aligned}
& \operatorname{Pr}\left[h_{o}>\gamma\left(\sum_{j \in \partial\left(n_{I}\right)} d_{j}(r, \theta)^{-\alpha} h_{j}\right)+\mathrm{A}\right] \\
& =\int_{h_{1}=0}^{\infty} \cdots \int_{h_{n_{I}=0}}^{\infty} \operatorname{Pr}\left[h_{o}>\gamma\left(\sum_{j \in \partial\left(n_{I}\right)} d_{j}(r, \theta)^{-\alpha} h_{j}\right)+\mathrm{A} \mid h_{1}, h_{2}, \ldots, h_{j}\right] \prod_{j=1}^{n_{I}} f_{j}\left(h_{j}\right) d h_{j} \\
& =e^{-\left(\frac{1}{\delta_{j}}\right) \mathrm{A}} \int_{h_{n_{I}=0}}^{\infty} \cdots \int_{h_{1}=0}^{\infty} e^{-\left(\frac{1}{\delta_{j}}\right) \gamma\left(\sum_{j \in \sigma\left(n_{I}\right)} d_{j}(r, \theta)^{-\alpha} h_{j}\right)} \prod_{j=1}^{n_{I}} f_{j}\left(h_{j}\right) d h_{j},
\end{aligned}
$$


where $f_{j}\left(h_{j}\right)$ denotes the PDF of $h_{j}$, i.e., $f_{j}\left(h_{j}\right)=\left(1 / \delta_{j}\right) e^{-\left(1 / \delta_{j}\right) h_{j}}$. When we integrate Eq. (22) with respect to $h_{j}$ first, we obtain

$$
e^{-\left(\frac{1}{\delta_{o}}\right) \mathrm{A}} \int_{h_{n_{I}=0}}^{\infty} \cdots \int_{h_{2}=0}^{\infty} e^{-\left(\frac{1}{\delta_{o}}\right) \gamma\left(\sum_{j \in \delta\left(n_{I}-1\right)} d_{j}(r, \theta)^{-\alpha} h_{j}\right)} \frac{1}{\frac{\delta_{1}}{\delta_{0}} \gamma d_{1}(r, \theta)^{-\alpha}+1} \prod_{j=2}^{n_{I}} f_{j}\left(h_{j}\right) d h_{j},
$$

where $\int_{0}^{\infty} e^{-G x} d x=1 / G$ is used. We can simplify (c) in Eq. (18) as follows:

$$
(\mathrm{c})=\prod_{j=1}^{n_{I}} \frac{e^{-\left(\frac{1}{\delta_{o}}\right) \gamma \sigma^{2} P_{t}^{-1} r_{o}(r, \theta)^{\alpha}}}{\delta_{j}} \gamma d_{j}(r, \theta)^{-\alpha}+1 .
$$

Accordingly, Eq. (19) can be rewritten as

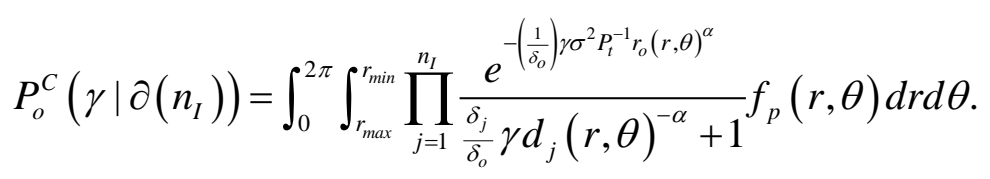

By using the total probability law for Eq. (20), we can obtain $P_{o}(\gamma)$ as follows:

$$
\begin{aligned}
P_{o}(\gamma) & =1-P_{o}^{C}(\gamma) \\
& =1-\sum_{n_{I}=0}^{18} \sum_{n_{I_{-} i}=0}^{n_{I}} \sum_{n_{I_{-} o}=0}^{n_{I}-n_{I_{-} i}} P_{o}^{C}\left[\gamma \mid \partial\left(n_{I}\right)\right] \operatorname{Pr}\left[\partial\left(n_{I}\right): n_{I_{-}}, n_{I_{-} o}\right] .
\end{aligned}
$$

Based on Eq. (14) and (26), once the outage probability of the inner UE using the inner PRB, $P_{o_{-} i i}(\gamma)$ and that of the inner UE using outer PRB, $P_{o_{-} i o}(\gamma)$, are obtained, we have

$$
P_{o i}(\gamma)=v_{i} P_{o_{-} i i}(\gamma)+\left(1-v_{i}\right) P_{o_{-} i o}(\gamma) \text {. }
$$

Once the outage probability of the inner UE, $P_{o i}$, and that of the outer UE, $P_{o o}$, respectively, are obtained, we can obtain the total outage probability, $P_{o}$, as follows:

$$
P_{o}=\frac{\lambda_{i} P_{o i}+\lambda_{o} P_{o o}}{\lambda}
$$




\section{Numerical Results}

From the previous section, we obtained an analytical model as a function of $\vartheta$ and $z$. Here, we will analyze the performance of the S-SFR-based system and compare it with the simulation results to verify its accuracy. Additionally, we will compare the performance of our scheme with other SFR schemes. The system parameters are chosen as shown in Table 2. It is noteworthy that in the figures, the lines are obtained from the equations given in the performance analysis, whereas the symbols represent the corresponding simulation results.

Table 2. System parameters

\begin{tabular}{|c|c|}
\hline Parameter & Value \\
\hline \hline Transmission power $\left(P_{t}\right)$ & $40 \mathrm{~W}$ \\
\hline Operating bandwidth & $10 \mathrm{MHz}$ \\
\hline Number of PRBs $(C)$ & 48 \\
\hline Path loss exponent $(\alpha)$ & 4.5 \\
\hline Thermal noise density & $-174 \mathrm{dBm} / \mathrm{Hz}$ \\
\hline Cell radius $(R)$ & $750 \mathrm{~m}$ \\
\hline Mean of exponential PDF $\left(1 / \delta_{j}\right)$ & 1 \\
\hline Outage threshold $(\gamma)$ & $-10 \mathrm{~dB}$ \\
\hline
\end{tabular}

\subsection{Traffic Performance}

In Fig. 4, 5 and 6, we investigate the traffic performances, such as the call blocking probability, the forced termination probability and the resource utilization. Generally, these numerical examples show that our analytical results are very similar to those of the simulations.
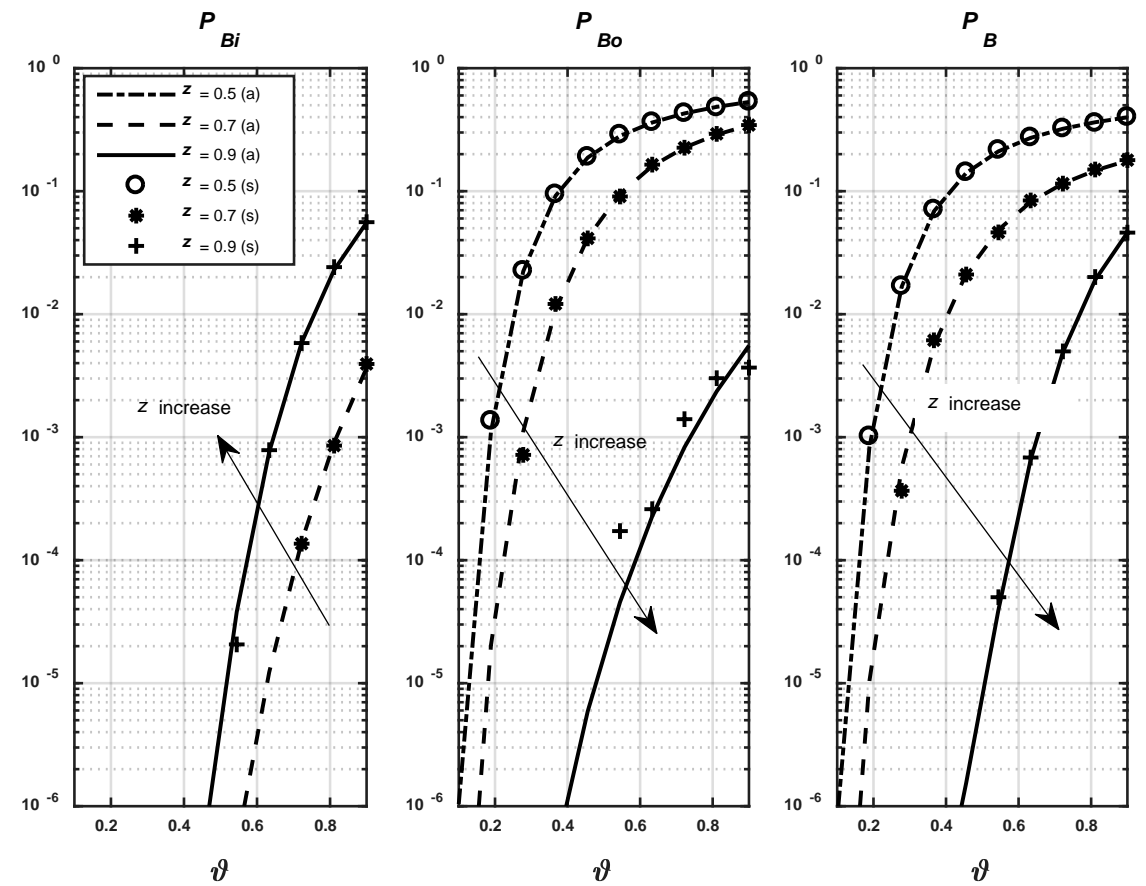

Fig. 4. Call blocking probability vs. offered load with various $Z$ 


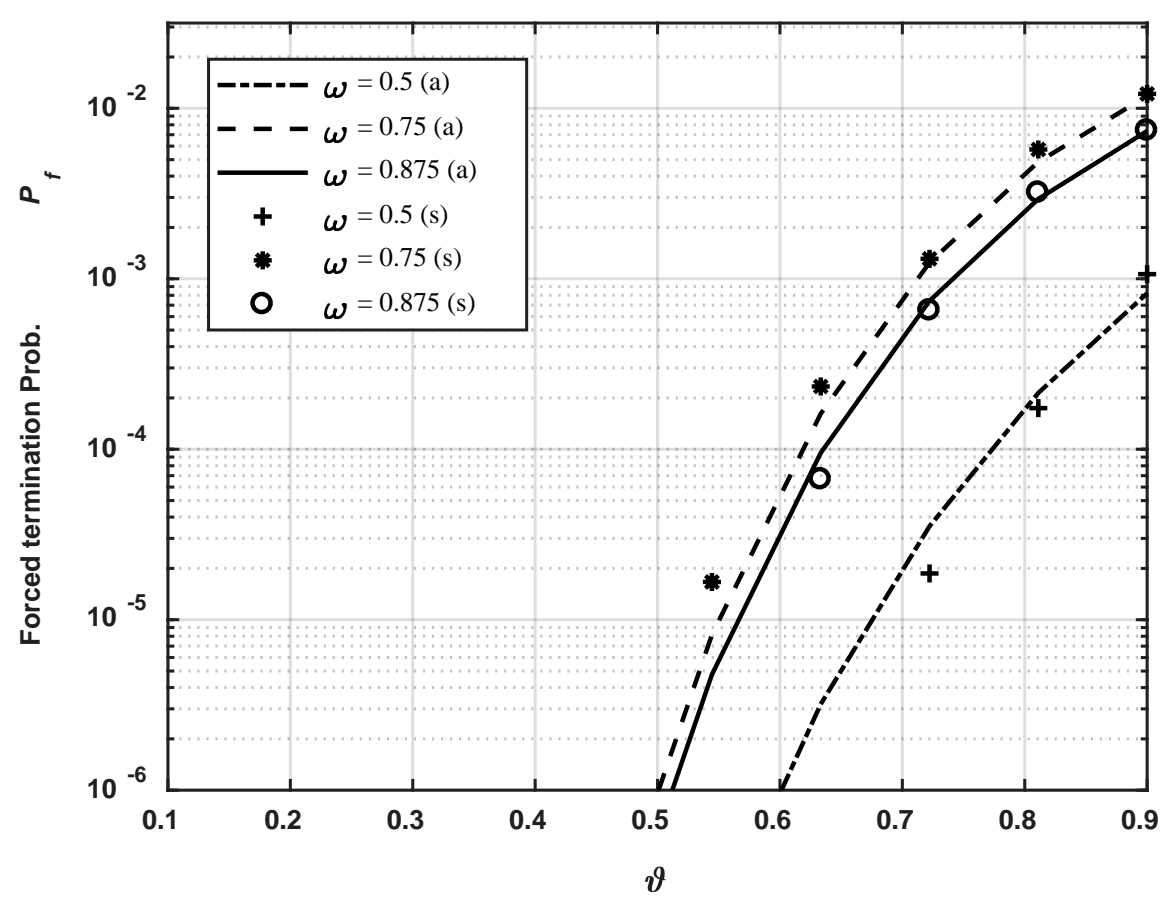

Fig. 5. Forced termination probabilities vs. offered load with various $\omega$
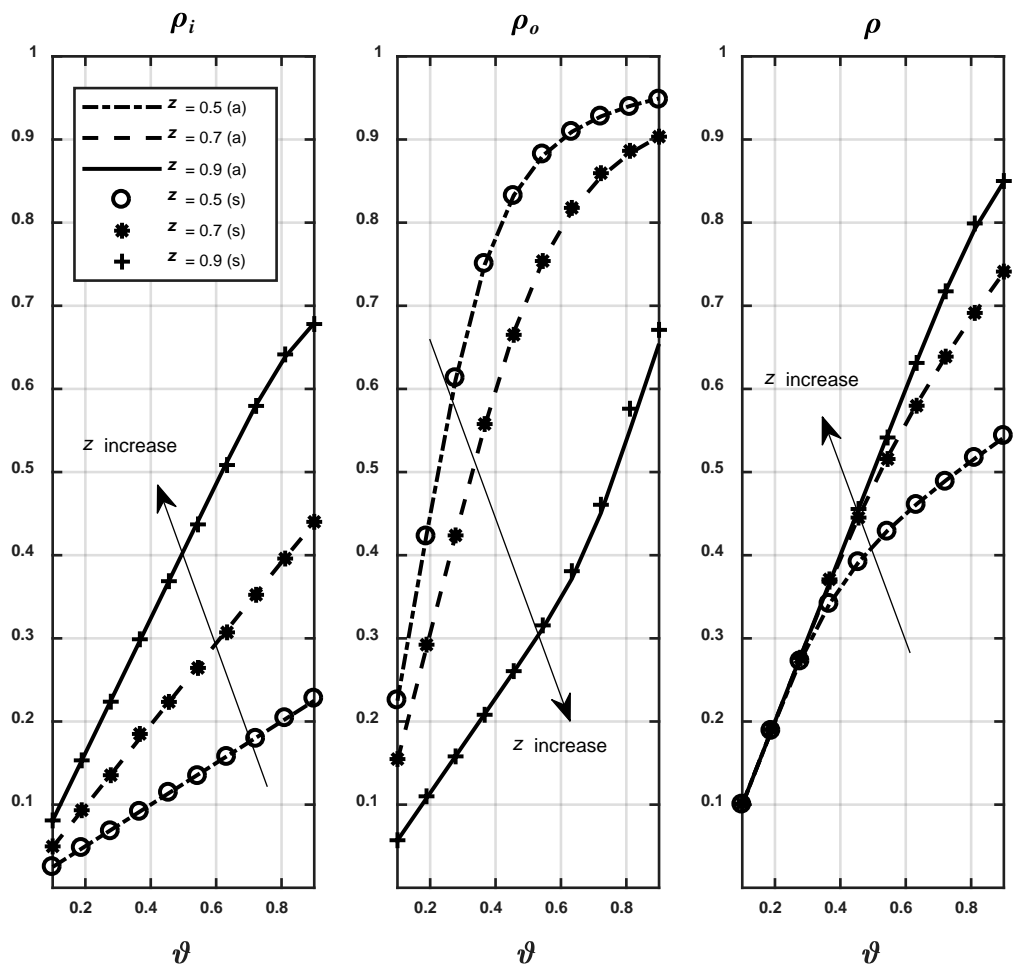

Fig. 6. Utilization vs. offered load with various $Z$ 
Fig. 4 shows the call blocking probabilities for various offered loads, $\vartheta$. For the effect of $z$, as this value increases, $P_{B o}$ and $P_{B}$ tend to decrease, whereas $P_{B i}$ increases, i.e., the blocking probabilities of the inner and outer are not evenly blocked. This is because as $Z$ increases, the number of UE reaching the outer region decreases; so, the number of blocked outer calls decreases. For $z=0.5$, as $P_{B i}$ is very small, this value is not shown in this figure. For $z=0.7$ and $\varepsilon_{B}=1 \times 10^{-2}, P_{B i}$ and $P_{B o}$ are satisfied at $\vartheta \leq 0.7$ and 0.2 , respectively.

The forced termination probability is plotted in Fig. 5. For $\vartheta, P_{f}$ is very small. As shown, $P_{f}$ increases as $\omega$ increases. This is because as $\omega$ increases, the number of inner UE increases, resulting in a decrease in the number of the inner UE that terminate.

Fig. 6 shows the resource utilizations for various offered loads, $\vartheta$. As shown in the figure, the resource utilizations are not balanced. It is noteworthy that, in Eq. (16) and (17), $\rho_{i}, \rho_{o}$ and $\rho$ depend on $Z$. As mentioned earlier, this is because $Z$ affects the number of UE arriving in each region. The large $z$ provides a large inner zone and a higher $\rho_{i}$, while $\rho_{o}$ decreases. The outer PRBs are shown to be more heavily utilized than the inner PRBs.

\subsection{Outage performance and system throughput}

Fig. 7, 8 and 9 show the outage probability and the system throughput of the proposed scheme. In general, based on these figures, our analysis agrees well with the simulations.
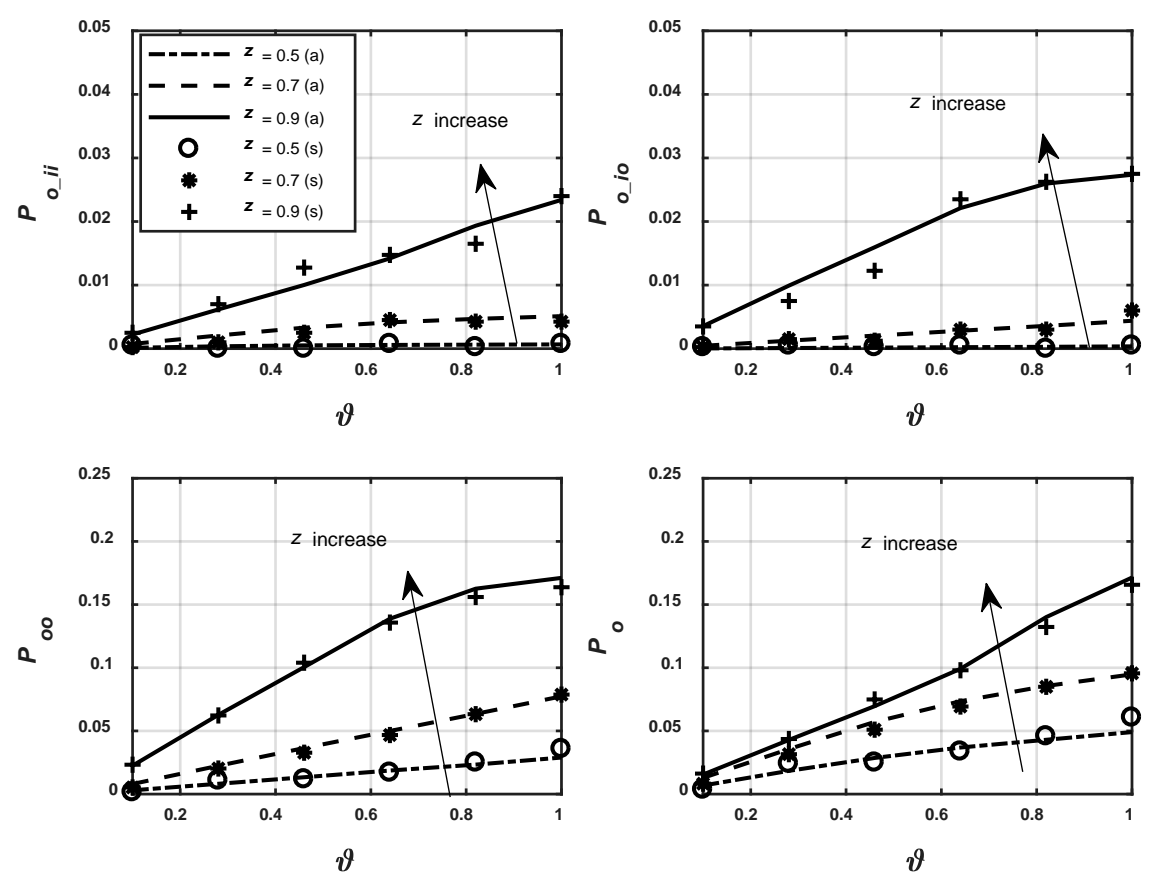

Fig. 7. Outage probability vs. offered load with various $Z$

Fig. 7 shows the outage probabilities for various offered loads, $\vartheta$. The results showed a significant disparity in the outage probability depending on the type of UE, where $P_{o_{-} i i}$ and 
$P_{o_{-} \text {io }}$ are lower than $P_{\text {oo }}$. as the inner UE are relatively close to the serving eNB. For the effect of $Z$, as $Z$ increases, the outage probability of each UE increases. This is because, as expected from the resource utilization result, an increased inner zone increases $\rho$ and leads to the extended probability that an interference occurs from the neighboring cells. For $z=0.7$ and $\varepsilon_{o}=0.1, P_{o}$ is satisfied at $\vartheta \leq 0.6$.

Fig. 8 shows the system throughput according to the values of various $Z$. In addition, this figure presents the result of Eq. (3). Fig. 8 also shows that the achievable system throughput depends not only on the offered load but also on the size of the inner area. As shown, at a lower $\vartheta$, each $S_{t h}$ is similar for different values of $Z$. Whereas as $Z$ increases, $S_{t h}$ increases at higher $\vartheta$.

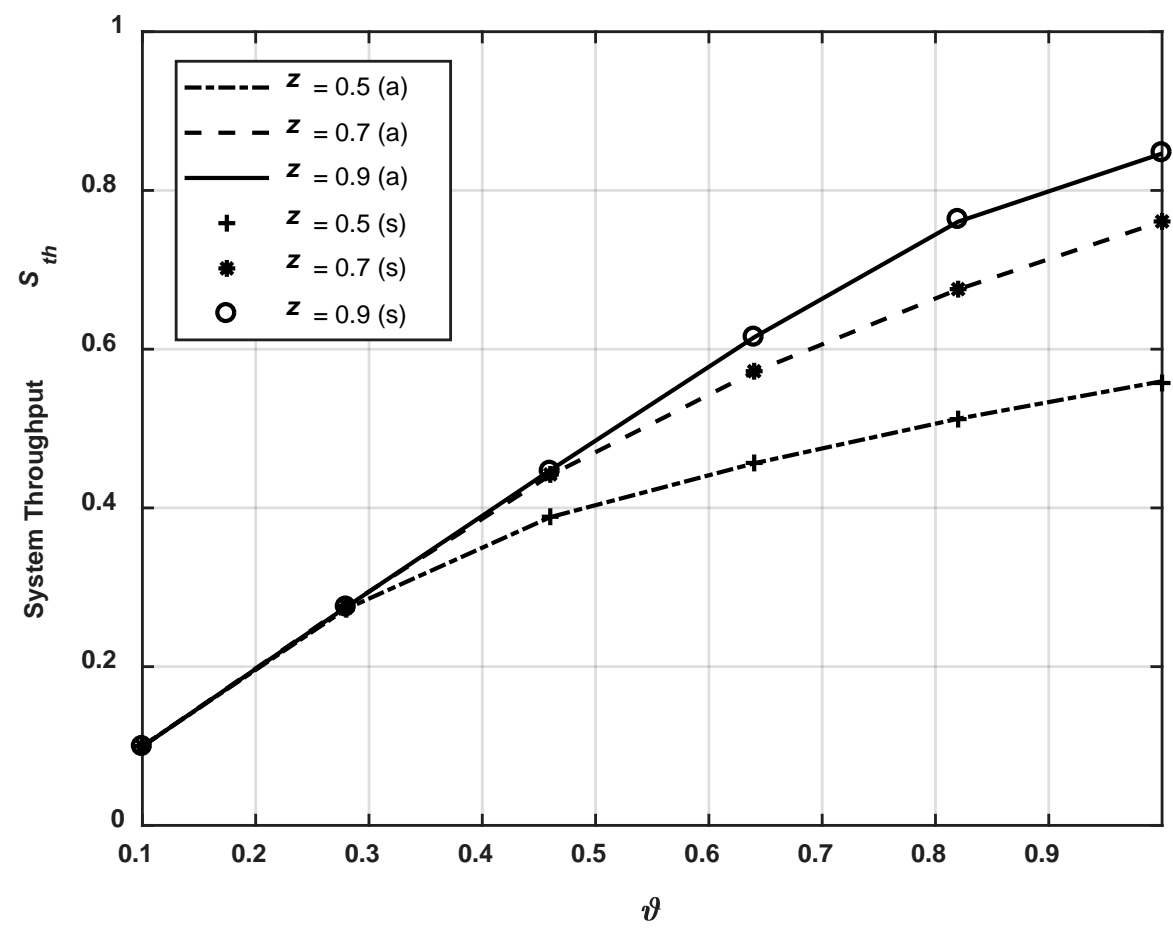

Fig. 8. System throughput vs. offered load with various $Z$

The relationship between the system throughput and the outage probability is plotted in Fig. 9. Also, Fig. 9 presents the result of Eq. (4). In this figure, we confirm that the system throughput cannot be increased without increasing $\varepsilon_{o}$. For example, when $\varepsilon_{o}=0.049$ and $Z=0.5$, the system throughput is 0.56 . And when $\varepsilon_{o}=0.095$ and $z=0.7$, the system throughput is 0.76 . However, for low outage probabilities (i.e., outage probability $<0.05$ ), the system throughput is less than 0.6.

Table 3. Summary of various schemes

\begin{tabular}{|c|c|c|c|}
\hline & S-SFR & SFR v2 & SFR v1 \\
\hline \hline Spectrum handoff & $\bigcirc$ & $X$ & $X$ \\
\hline Preemption & $\bigcirc$ & $\bigcirc$ & $X$ \\
\hline
\end{tabular}


Additionally, we compare the performances of our scheme with other ICIC schemes. In Fig. 10, SFR v1 is the scheme shown in Fig. 1 and SFR v2 is the proposed scheme in [7]. In [7], similar to our proposed scheme, an inner UE can use an outer PRB and be forced terminated by an outer UE, whereas an inner UE cannot use the spectrum handoff. Each scheme is summarized in Table 3.

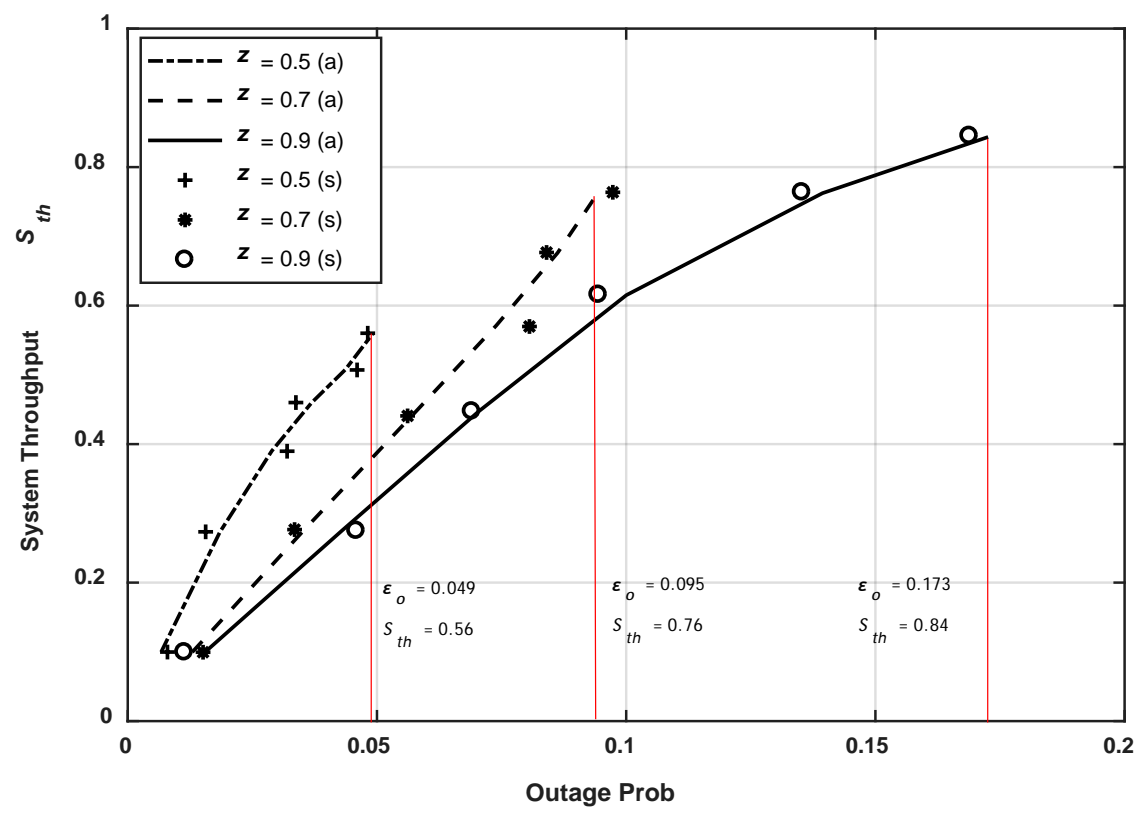

Fig. 9. System throughput vs. outage probability with various $Z$

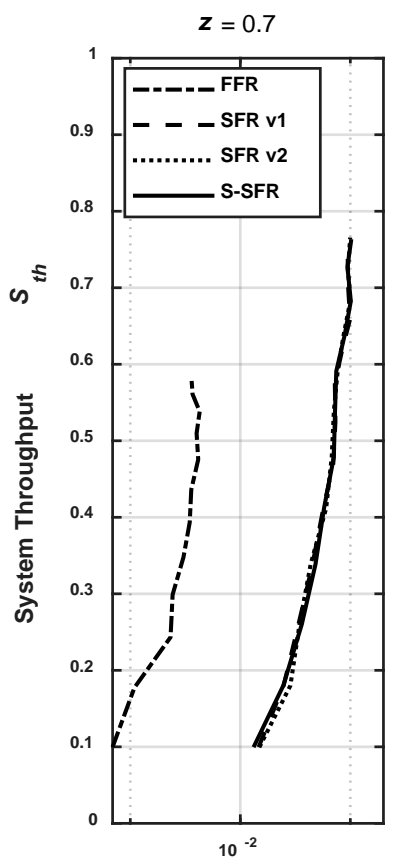

Outage Prob.

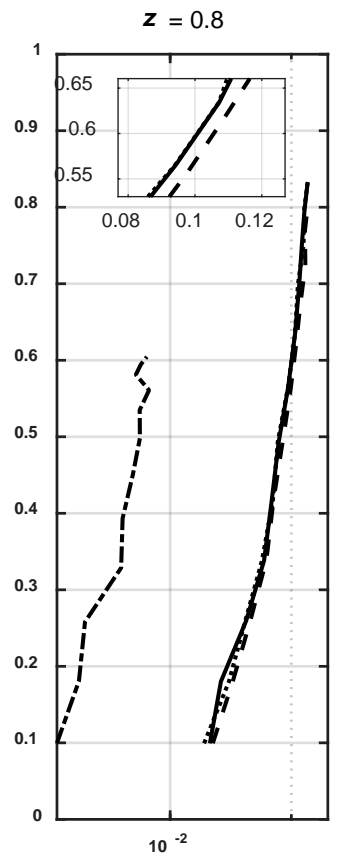

Outage Prob.

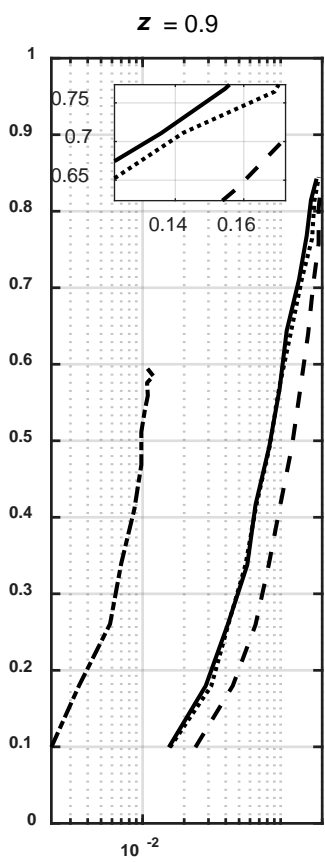

Outage Prob.

Fig. 10. System throughput vs. offered load with various $Z$ and schemes 
Fig. 10 shows that at a lower $\varepsilon_{o}$, the system throughput of the FFR scheme can achieve the maximum value. Whereas for increased $\varepsilon_{o}$, the SFR-based system can obtain an increased system throughput. This is because, in FFR, some outer PRB is unused, thus leading to the increase in the call blocking probability and the decrement of system utilization. For an SFR-based system, we note that the PRB assignment of the inner UE using all PRBs is more efficient than that of the inner UE using the limited PRB set. In this figure, we observed that our scheme outperforms the other SFR schemes.

\section{Conclusion}

In this paper, to overcome the problems of the existing SFR schemes, we proposed the analysis model for S-SFR-based OFDMA cellular system. We analyzed the proposed system through performance indicators of outage probability, call blocking probability, system throughput and resource utilization.

In the analytical model, we first used a 2-D Markov chain analysis for traffic performance to obtain the resource utilization and the call blocking probability. Also, we have obtained the closed-form expression of the outage probability, where resource utilization is used for the probability of interference occurring. Finally, the system throughput is derived using the outage probability and call blocking probability. We showed that our analysis results are consistent with most of the simulation results. The results showed that the size of the inner area affects the system performance. The significant result also was found that the system throughput achieves its peak at $z=0.7$. A tradeoff is observed between the system throughput and outage probability.

Additionally, we compared our scheme with other ICI schemes. The result showed that limited PRB assignment can improve outage performance while deteriorating the system throughput.

\section{References}

[1] E. Dahlman, S. Parkvall and J. Skold, 4G: LTE/LTE-Advanced for Mobile Broadband, 2nd Edition, Elsevier Academic Press, Oxford, 2013. Article (CrossRef Link)

[2] Eunsung Oh, Myeon-Gyun. Cho, Seungyoup Han, Choongchae Woo and Daesik Hong, "Performance Analysis of Reuse-Partitioning-Based Subchannelized OFDMA Uplink Systems in Multicell Environments," IEEE Transactions on Vehicular Technology, vol. 57, no. 4, pp. 2617-2621, 2008. Article (CrossRef Link)

[3] B. Soret, A. D. Domenico, S. Bazzi, N. H. Mahmood and K. I. Pedersen, "Interference Coordination for 5G New Radio," IEEE Wireless Communications, to be published, Early Access 2018. Article (CrossRef Link)

[4] M. Yassin, M. A. AboulHassan, S. Lahoud, M. Ibrahim, D. Mezher and B. Cousin, "Survey of ICIC techniques in LTE networks under various mobile environment parameters," Wireless Networks, vol. 23, no. 2, pp. 403-418, 2017. Article (CrossRef Link)

[5] A. S. Hamza, S. S. Khalifa, H. S. Hamza and K. Elsayed, "A Survey on Inter-Cell Interference Coordination Techniques in OFDMA-Based Cellular Networks," IEEE Communications Surveys \& Tutorials, vol. 15, no. 4, pp. 1642-1670, 2013. Article (CrossRef Link)

[6] A. Mahmud and A. Hamdi, "A unified framework for the analysis of fractional frequency reuse techniques," IEEE Transactions on Communications, vol. 62, no. 10, pp. 3692-3705, 2014. Article (CrossRef Link) 
[7] S. P. Chung and Y. W. Chen, "Performance Analysis of Call Admission Control in SFR-Based LTE Systems,” IEEE Communications Letters, vol. 16, no. 7, pp. 1014-1017, 2012. Article (CrossRef Link)

[8] M. Yassin, S. Lahoud, M. Ibrahim, K. Khawam, D. Mezher and B. Cousin, "Non-cooperative inter-cell interference coordination technique for increasing throughput fairness in LTE networks," in Proc. of IEEE Vehicular Technology Conference (VTC-Spring), pp. 1-5, July 11-14, 2015. Article (CrossRef Link)

[9] 3rd Generation Partnership Project(3GPP) TS 36.213 V11.11.0, "Evolved universal terrestrial radio access (EUTRA): Physical layer procedures (Release 11),” July 2015. Article (CrossRef Link)

[10] E. Hossain, M. Rasti, H. Tabassum and A. Abdelnasser, "Evolution toward 5G multi-tier cellular wireless networks: An interference management perspective,” IEEE Wireless Communications, vol. 21, no. 3, pp. 118-127, 2014. Article (CrossRef Link)

[11] R. Mendrzik, R. A. J. Castillo, G. Bauch and E. Seidel, "Interference coordination-based downlink scheduling for heterogeneous LTE-A networks," in Proc. of IEEE Wireless Communications and Networking Conference (WCNC), pp. 1-6, April 3-6, 2016. Article (CrossRef Link)

[12] T. Novlan and J. Andrews, “Analytical evaluation of uplink fractional frequency reuse," IEEE Transactions on Communications, vol. 61, no. 5, pp. 2098-2108, 2013. Article (CrossRef Link)

[13] H. Zhu and J. Wang, "Performance Analysis of Chunk-Based Resource Allocation in Multi-Cell OFDMA Systems,” IEEE Journal on Selected Areas in Communications, vol. 32, no. 2, pp. 367-375, 2014. Article (CrossRef Link)

[14] J. Huang, J. Li, L. Zhao and S. Huang, "CoSFR: coordinated soft frequency reuse for OFDMA-based multi-cell networks with non-uniform user distribution,” Wireless Networks, vol. 23, no. 7, pp. 2037-2050, 2017. Article (CrossRef Link)

[15] J. Garcia-Morales, G. Femenias and F. Riera-Palou, “On the Design of OFDMA-based FFR-aided Irregular Cellular Networks with Shadowing,” IEEE Access, vol. 6, pp. 7641-7653, 2018. Article (CrossRef Link)

[16] S. Kumar, S. Kalyani and K. Giridhar, "Optimal design parameters for coverage probability in fractional frequency reuse and soft frequency reuse,” IET Communications, vol. 9, no. 10, pp. 1324-1331, 2015. Article (CrossRef Link)

[17] S. C. Lam and K. Sandrasegaran, "Analytical coverage probability of a typical user in heterogeneous cellular networks,” Journal of Networks, vol. 12, no.2, pp. 56-61, 2016. Article (CrossRef Link)

[18] L. Huo, D. Jiang and Z. Lv, "Soft frequency reuse-based optimization algorithm for energy efficiency of multi-cell networks,” Computers and Electrical Engineering, vol. 66, pp. 316-331, 2018. Article (CrossRef Link)

[19] M. S. Hossain, F. Tariq and G. A. Safdar, "Enhancing cell-edge performance using multi-layer soft frequency reuse scheme,” Electronics Letters, vol. 51, no. 22, pp. 1826-1828, 2015. Article (CrossRef Link)

[20] Seung-Yeon Kim, Hyong-Woo Lee and Choong-Ho Cho, “Analytical Evaluation of Call Admission Control for SFR-Based LTE Systems," in Proc. of International Conference on Networking and Services, pp. 69-73, April 20-24, 2014. Article (CrossRef Link)

[21] Mustafa H. Mohsini, Seung-Yeon Kim and Choong-Ho Cho, "Throughput and Power Efficiency Analysis Considering Traffic Loads in FFR with Movable Boundary,” IETE Journal of Research, to be published, Early Access 2018. Article (CrossRef Link)

[22] Sok Chhorn, Seok-Ho Yoon, Si-O Seo, Seung-Yeon Kim and Choong-Ho Cho, "Spectrum Reuse Schemes with Power Control for Device-to-Device Communication in LTE-Advanced Cellular Network," KSII Transactions on Internet and Information Systems, vol. 9, no. 12, pp. 4819-4834, 2015. Article (CrossRef Link)

[23] J. Ryu and D. Cho, “A new routing scheme concerning power-saving in mobile ad-hoc networks," in Proc. of IEEE International Conference on Communications (ICC), pp. 1719-1722, June 18-22, 2000. Article (CrossRef Link) 
[24] S. U. Abdullahi, J. Liu and S. A. Mohadeskasaei, "Analytical Evaluation of FFR-aided Heterogeneous Cellular Networks with Optimal Double Threshold," KSII Transactions on Internet and Information Systems, vol. 11, no. 7, pp. 3370-3392, 2017. Article (CrossRef Link)

[25] J. Garcia-Morales, G. Femenias and F. Riera-Palou, "Statistical Analysis and Optimization of a Fifth-Percentile User Rate Constrained Design for FFR/SFR-Aided OFDMA-Based Cellular Networks,” IEEE Transactions on Vehicular Technology, vol. 67, no. 4, pp. 3406-3419, 2018. Article (CrossRef Link)
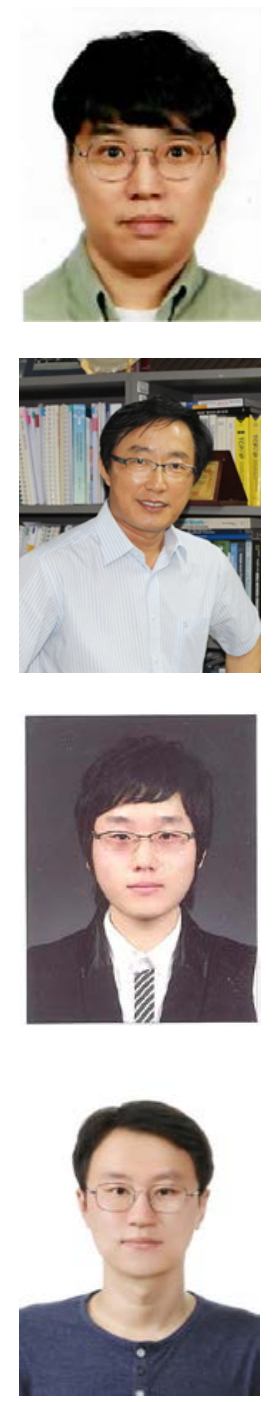

Yi-Kang Kim received his master's degree in computer science in 2010 from Korea University, Korea. He is currently a $\mathrm{PhD}$ candidate in the Department of Computer Convergence Software at Korea University. His research interests include performance evaluation of communication networks, interference management, 5G network protocol for mobile edge computing, IoT, and SDN/NFV.

Choong-Ho Cho received his $\mathrm{PhD}$ degree in computer science in 1989 from INSA de Lyon University, France. He is currently a professor at Korea University in the Department of Computer Convergence Software since 1994. He has participated in various research projects involving an IMT-advanced system, cognitive radio, interference management, energy management systems, and heterogeneous networks. His research interests include performance evaluation of communication networks, energy efficiency, 4G/5G mobile/wireless networks, internet business, ICT convergence, building/home energy management system, smart city platform.

Seok-Ho Yoon received the B.S and M.S degrees in Computer and Information Science in 2009 and 2011, respectively, from Korea University, Korea, where he is currently working toward the Ph.D. degree in the Laboratory for information \& communication convergence technology, Korea University. He has participated in various research projects involving an interference management, BEMS (building energy management system), demand response, design and analysis of energy management systems.

Seung-Yeon Kim received his $\mathrm{PhD}$ degree in electronics and information engineering in 2012 from Korea University, Korea. He is currently an assistant professor in the Department of Computer Convergence Software at Korea University. He has participated in various research projects involving an IMT-advanced system, cognitive radio, and interference management. His research interests include performance evaluation of communication networks. 